\title{
Establishment of logic gates based on conformational changes in multiple-factor biomolecule interaction process by dual polarization interferometry
}

Shuang Wang, ${ }^{\dagger+\ddagger}$ Jiahui Zhao, ${ }^{\dagger, \S}$ Shasha Lu, ${ }^{\dagger, \downarrow}$ Jianshe Huang ${ }^{*, \dagger}$ and Xiurong Yang ${ }^{*},+, \ldots, \S$

$\dagger$ State Key Laboratory of Electroanalytical Chemistry, Changchun Institute of Applied Chemistry,

Changchun, Jilin 130022, China

$\$$ University of Science and Technology of China, Hefei, Anhui 230026, China

$\S$ University of Chinese Academy of Sciences, Beijing 100039, China

${ }^{*}$ Corresponding author. Tel.: +86 431 85262056; fax: +86 43185689278.

E-mail address: xryang@ciac.ac.cn; huangjs@ciac.ac.cn.

\section{Content}

Experimental section $\quad 2-4$

Fig. S1. CD characterization for the interaction between ssDNA and metal ions. 5

Table S1. Structural parameters of different layer materials. 5

$\begin{array}{ll}\text { Fig. S2. The whole structural transition process of PEI/AS1411/ } / \mathrm{K}^{+} / \mathrm{Hg}^{2+} / \mathrm{Cys} . & 6\end{array}$

$\begin{array}{ll}\text { Fig. S3. The density and thickness changes as the mass changes. } & 6\end{array}$

Fig. S4. Construction of IDENTITY logic gate based on $\mathrm{K}^{+}$and $\mathrm{Pb}^{2+}$.

Fig. S5. Construction of IDENTITY logic gate based on $\mathrm{K}^{+}$and $\mathrm{Hg}^{2+}$.

Fig. S6. Reversibility evaluation of the proposed system. 8 


\section{Experimental section}

Material. Poly(ethyleneimine) solution (PEI, 50\% in $\mathrm{H}_{2} \mathrm{O}, \mathrm{MW} 750000$ ) and Mercury( II ) chloride $\left(\mathrm{HgCl}_{2}\right)$ were obtained from Sigma-Aldrich (St Louis, MO). Tris(hydroxymethyl) aminomethane (Tris, ultrapure grade) was provided from Amresco (Shanghai, China). Acetic acid (Ac) and Lead (II) acetate trihydrate $\left[\mathrm{Pb}(\mathrm{Ac})_{2} \cdot 3 \mathrm{H}_{2} \mathrm{O}\right]$ were obtained from Aladdin Chemistry Co. Ltd (St Louis, MO). Potassium chloride $(\mathrm{KCl})$ was purchased from Beijing Chemical Reagent Co. (Beijing, China). Cysteine was purchased from Beijing Dingguo Biotechnology Co. (Beijing, China). N-methyl mesoporphyrin IX (NMM) were purchased from Sigma-Aldrich (St Louis, MO). SYBR Green II was obtained from Beijing Solarbio Science \& Technology Co., Ltd. Ultrapurewater (Milli-Q synthesis, Millipore, Inc., Bedford, MA) was used throughout. Tris-Ac buffer solution $(\mathrm{pH}=7.4)$ was prepared with $10 \mathrm{mM}$ Tris and adjusted by acetic acid. The buffer solution should be filtered and degassed before use. 26-mer guanine-rich ssDNA oligonucleotides AS1411 containing mercury-specific sequences were synthesized by Sangon Biotechnology Co. Ltd. (Shanghai, China). Before use, oligonucleotides were dissolved in Tris-Ac buffer ( $\mathrm{pH}$ 7.4) as stock solutions, and the concentrations were quantified by measuring the UV absorption at $260 \mathrm{~nm}$. The stock solutions were stored in a refrigerator at $4^{\circ} \mathrm{C}$ before use. All chemicals were used without further purification or treatment. Unless otherwise mentioned, all work solutions were prepared with Tris-Ac buffer solution (10 mM, pH 7.4). The sequences of AS1411 and its control strand displayed below:

AS1411: GGTGGTGGTGGTTGTGGTGGTGGTGG;

Control strand: AAAAAAAAAAAAAAAAAAAAAAAAAA

\section{DPI Experiment.}

The structural transition among single strand, double helix and G-quadruplex was monitored in real-time utilizing a DPI instrument (AnaLight Bio200, Farfield Group 
Ltd., Crewe,U.K.). Unmodified silicon oxynitride AnaChip (FB 80, Farfield Scientific Ltd.) was used in all experiments. Before use, the chip was first cleaned overnight with Piranha solution (a 7:3 mixture of concentrated sulfuric acid and 30\% hydrogen peroxide). Subsequently, it was rinsed in ultrapure water under sonication for five times ( 6 min each time) and then dried under a stream of nitrogen to get rid of water from the surface. All experiments were performed at $20 \pm 0.002{ }^{\circ} \mathrm{C}$ in $10 \mathrm{mM}$ Tris-Ac running buffer solution $(\mathrm{pH}$ 7.4). After the cleaned chip was loaded into the instrument, the running buffer solution was passed over the chip at a flow rate of 100 $\mu \mathrm{L} / \mathrm{min}$. When the baseline was stable, an $80 \%$ (v/v) ethanol/water solution and ultrapure water with known refractive indexes were sequentially injected into the two channels to calibrate the chip and the running buffer. Thickness and refractive index of the top waveguide layer and the refractive index of the running buffer were obtained and applied for the following experiment data calculation. After calibration, $200 \mu \mathrm{L}$ PEI $(0.1 \mathrm{mg} / \mathrm{mL})$ was applied to both channels at a flow rate of $50 \mu \mathrm{L} / \mathrm{min}$, then incubated for $5 \mathrm{~min}$ before the running buffer returned to both channels. Subsequently, $15 \mu \mathrm{M}$ ssDNA was injected for $4 \mathrm{~min}$ and incubated for $5 \mathrm{~min}$, then the flow returned to the running buffer. When the stable baseline was reached, targets such as $\mathrm{K}^{+} / \mathrm{Pb}^{2+}, \mathrm{Hg}^{2+}$ and cysteine were injected for 4 min and incubated for $5 \mathrm{~min}$, respectively. The running buffer flowed over the chip until the stable baseline was attained. All the data were analyzed by the analysis software of the AnaLight Bio200 instrument and translated into refractive index, thickness, mass, and density values.

\section{Circular Dichroism (CD) Experiment.}

CD spectral measurement was performed on a Jasco J-820 circular dichroism spectropolarimeter (Tokyo, Japan). Prior to measurement, the buffer solution (10 mM Tris-Ac, $\mathrm{pH}$ 7.4) was scanned as the background, which would be automatically subtracted from the following CD data. Two hundred microliters of $10 \mathrm{mM}$ Tris-Ac buffer solution ( $\mathrm{pH} 7.4$ ) containing $6.25 \mu \mathrm{M}$ AS1411 (or control DNA) was mixed with different ions respectively. The mixtures were equilibrated for $40 \mathrm{~min}$ at room temperature and then were independently added into a cell made of quartz suprasil (10 
$\mathrm{mm}$ path length, $0.7 \mathrm{~mL}$ volume). The $\mathrm{CD}$ spectra were measured at room temperature over the wavelength range of 200-350 nm with a scanning speed of 200 $\mathrm{nm} / \mathrm{min}$. The ultimate reported spectrum was the average of three successive scans.

\section{Fluorescence Spectrometry Experiment}

Fluorescence spectra were measured on F-4600 FL spectrophotometer (Hitachi, Japan). $2 \mu \mathrm{M}$ AS1411 reacted with different metal ions combinations for $40 \mathrm{~min}$ at room temperature, then mixture incubated with $5 \mu \mathrm{M}$ NMM and 10x SYBR Green II for one hour, respectively. Finally, the fluorescence spectra were collected. The excitation/emission wavelengths of NMM and SYBR Green II were set at 399/610 nm, 497/520 nm, respectively. 

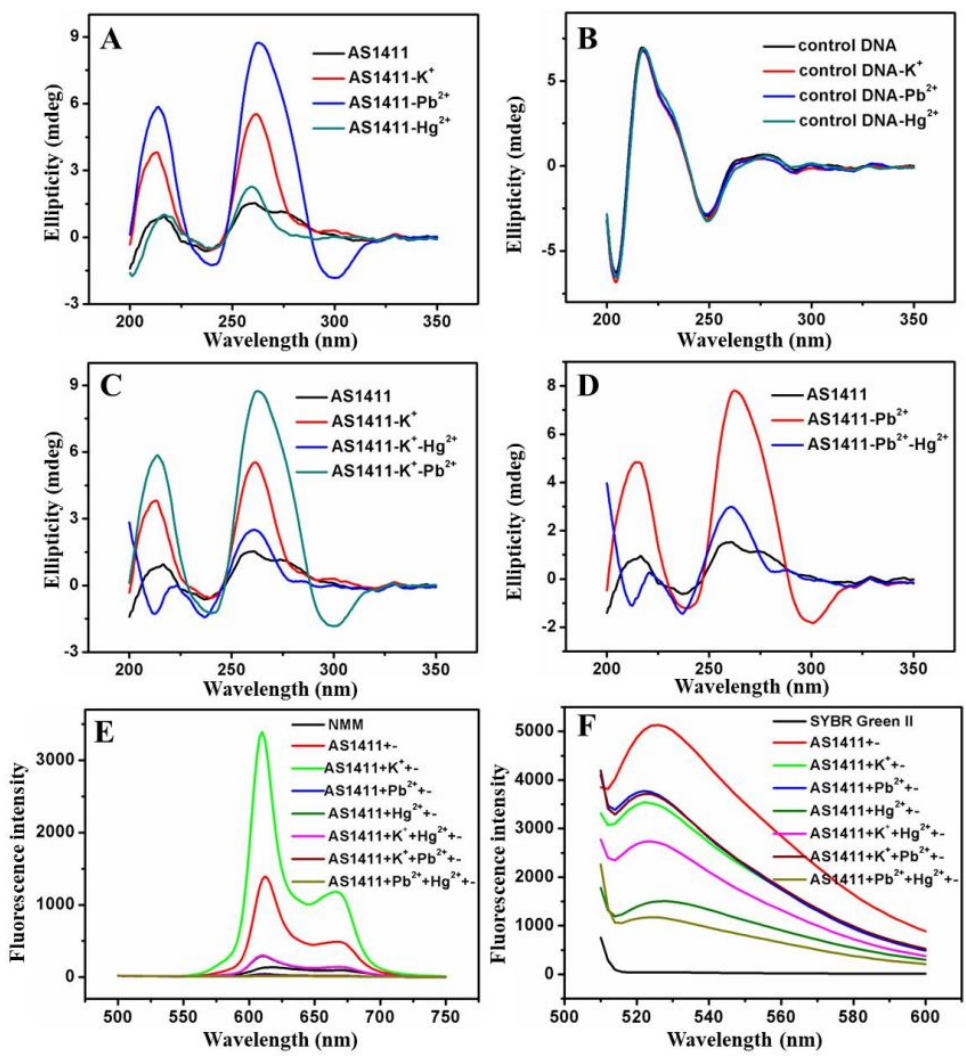

Fig. S1 CD spectra of AS411 (A) and control DNA (B) after the addition of different metal ions of $\mathrm{K}^{+}, \mathrm{Pb}^{2+}$ and $\mathrm{Hg}^{2+}$ in $10 \mathrm{mM}$ Tris-Ac buffer solution (pH 7.4). $\mathrm{CD}$ spectra of AS1411 after adding $\mathrm{K}^{+}, \mathrm{K}^{+}+\mathrm{Hg}^{2+}, \mathrm{K}^{+}+\mathrm{Pb}^{2+}(\mathrm{C})$ and $\mathrm{Pb}^{2+}, \mathrm{Pb}^{2+}+\mathrm{Hg}^{2+}(\mathrm{D})$. Fluorescent spectra of AS1411 reacting with different metal ions combinations after introducing NMM (E) and SYBR Green II (F).

Table S1. Layer Structure for PEI/AS1411/ $\mathrm{Pb}^{2+} / \mathrm{Hg}^{2+} /$ Cysteine (Mean $\pm \mathrm{SD}, \mathrm{n}=3$ )

\begin{tabular}{llll}
\hline Layer material & Thickness $(\mathrm{nm})$ & Mass $\left(\mathrm{ng} / \mathrm{mm}^{2}\right)$ & Density $\left(\mathrm{g} / \mathrm{cm}^{3}\right)$ \\
\hline PEI & $2.052 \pm 0.144$ & $1.632 \pm 0.166$ & $0.794 \pm 0.024$ \\
PEI/AS1411 & $3.252 \pm 0.317$ & $2.458 \pm 0.164$ & $0.757 \pm 0.023$ \\
$\mathrm{PEI} / \mathrm{AS} 1411 / \mathrm{Pb}^{2+}$ & $3.663 \pm 0.194$ & $2.794 \pm 0.089$ & $0.763 \pm 0.027$ \\
$\mathrm{PEI} / \mathrm{AS} 1411 / \mathrm{Pb}^{2+} / \mathrm{Hg}^{2+}$ & $3.681 \pm 0.251$ & $3.113 \pm 0.183$ & $0.846 \pm 0.008$ \\
$\mathrm{PEI} / \mathrm{AS} 1411 / \mathrm{Pb}^{2+} / \mathrm{Hg}^{2+} / \mathrm{Cysteine}$ & $3.625 \pm 0.028$ & $2.766 \pm 0.126$ & $0.764 \pm 0.024$ \\
\hline
\end{tabular}




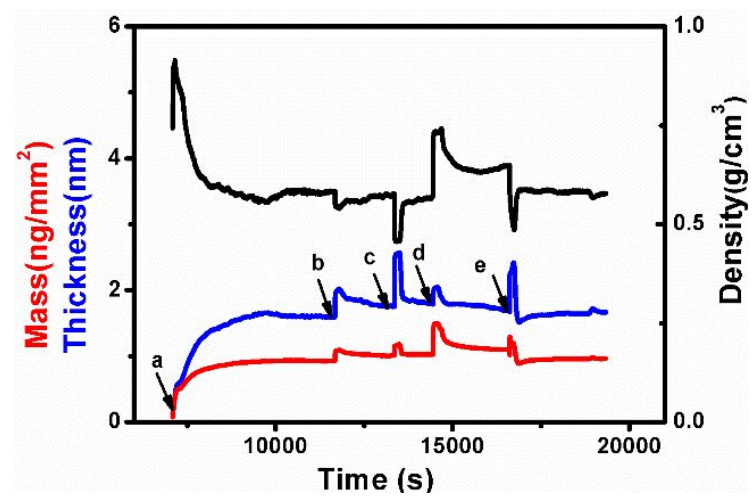

Fig. S2 DPI-based real-time measurements of mass (red color), thickness (blue color), and density (black color) for the whole immobilization process of PEI (a), AS1411 (b), and the interaction process with $\mathrm{K}^{+}$(c), $\mathrm{Hg}^{2+}$ (d) and cysteine (e) on the bare silicon oxynitride surface.
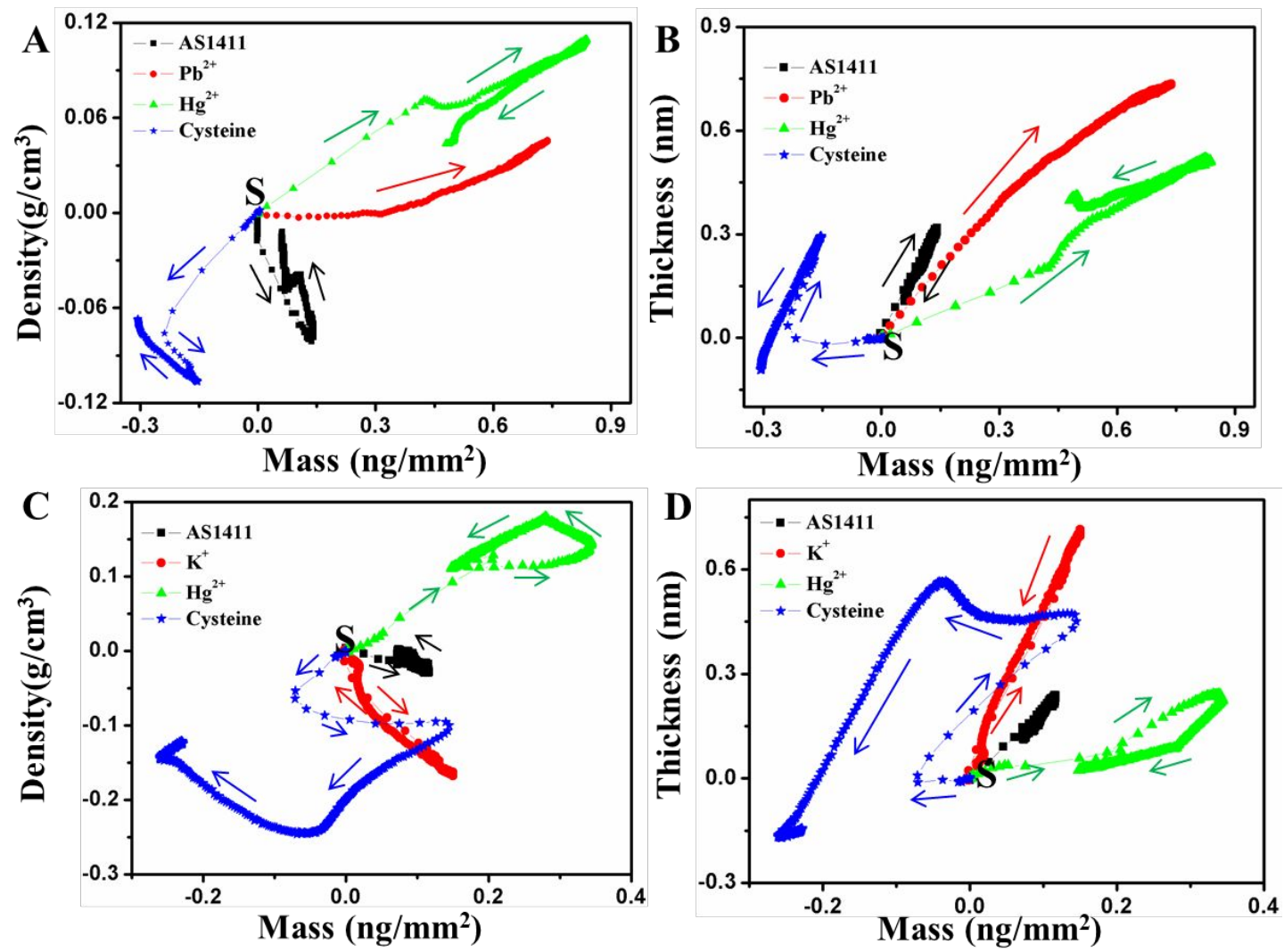

Fig. S3. Plot layer density (A) and thickness (B) of AS1411, $\mathrm{Pb}^{2+}, \mathrm{Hg}^{2+}$ and cysteine as a function of each respective mass loading during the entire process of each factor interaction. Plot layer density (C) and thickness (D) of AS1411, $\mathrm{K}^{+}, \mathrm{Hg}^{2+}$ and cysteine as a function of each respective mass loading during the entire process of each factor interaction. S represents starting point, and colored arrows point out the changed direction. 


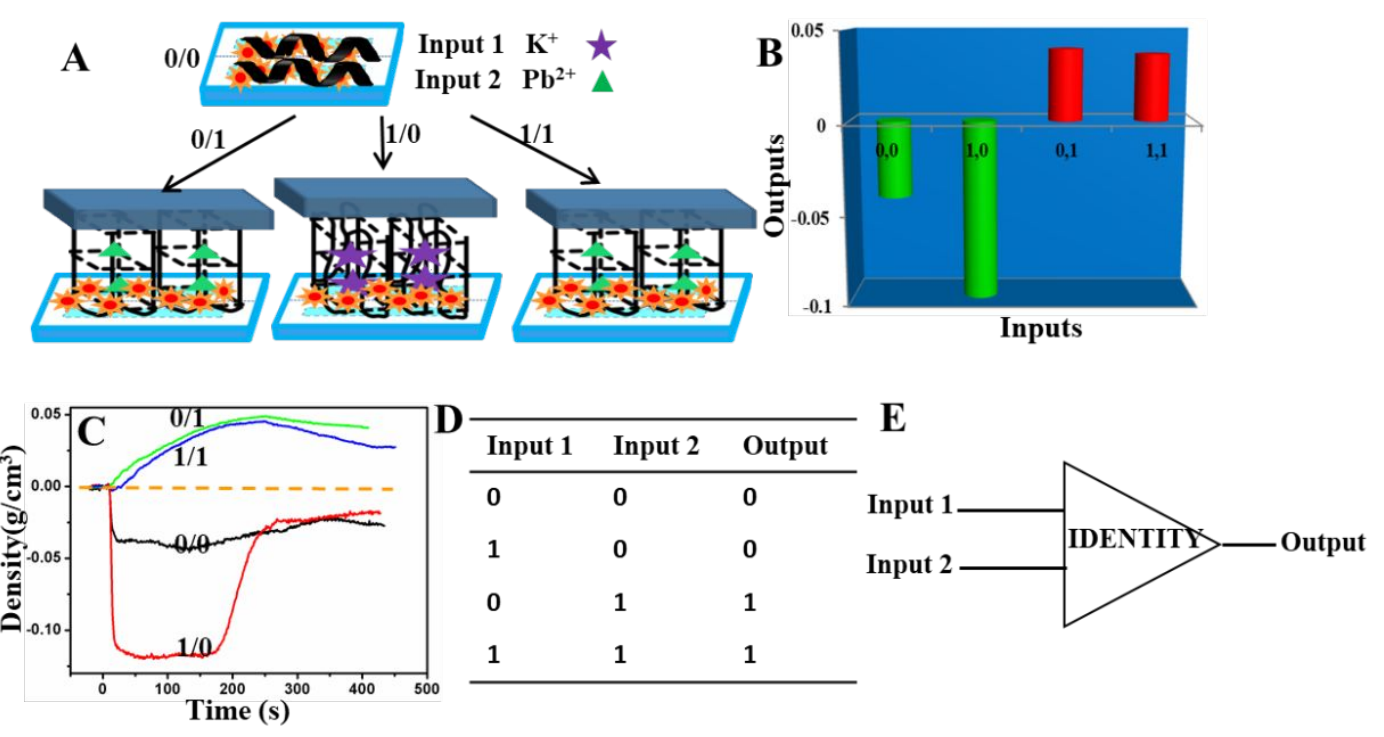

Fig. S4. Construction of IDENTITY logic gate based on $\mathrm{K}^{+}$and $\mathrm{Pb}^{2+}$. (A) Schematic illustration of the operational results of the logic gate. (B) The corresponding density changes measured by DPI when introduced different inputs. (C) The density changes histogram of different inputs. (The outputs corresponded to the density values recoded at $150 \mathrm{~s}$ in B.) (D) The truth table of the "IDENTITY" logic gate. (E) Electronic equivalent circuitry.
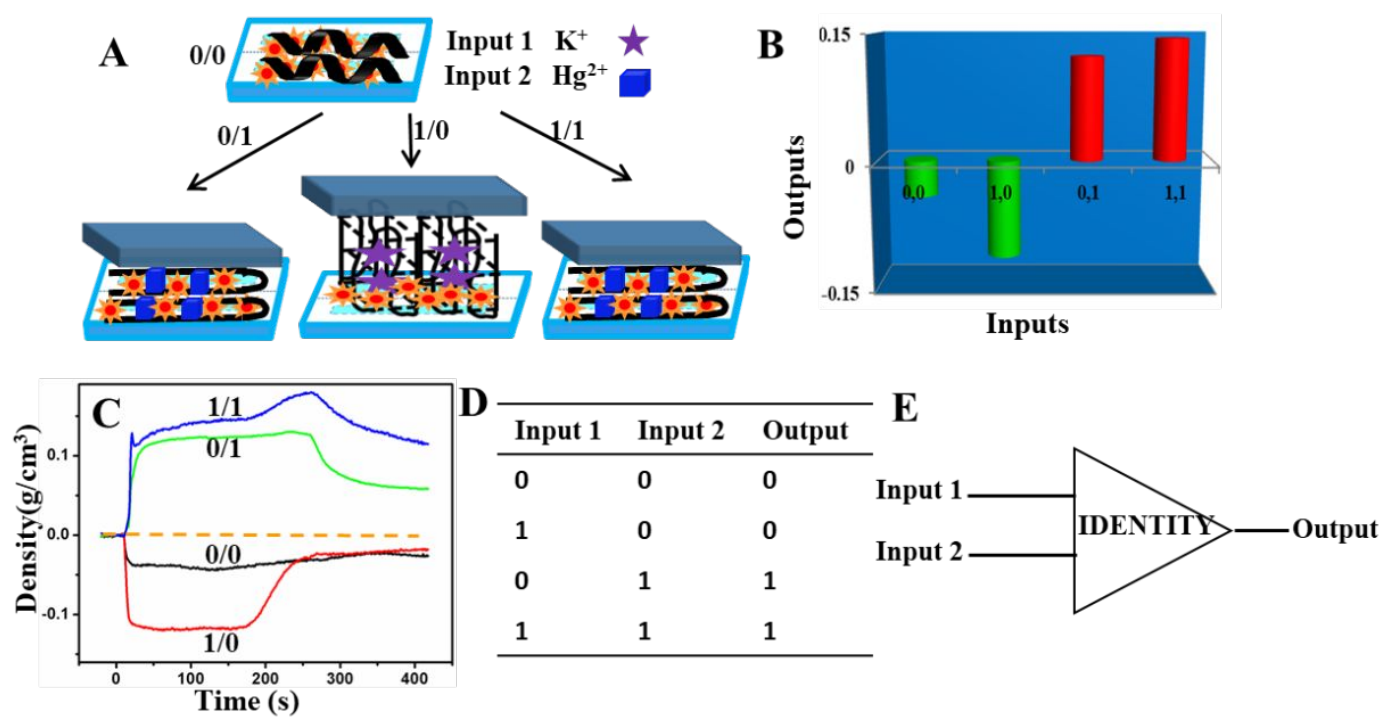

Fig. S5. Construction of IDENTITY logic gate based on $\mathrm{K}^{+}$and $\mathrm{Hg}^{2+}$. (A) Schematic illustration of the operational results of the logic gate. (B) The corresponding density changes measured by DPI when introduced different inputs. (C) The density changes histogram of different inputs. (The outputs corresponded to the density values recoded at $150 \mathrm{~s}$ in B.) (D) The truth table of the "IDENTITY" logic gate. (E) Electronic equivalent circuitry. 


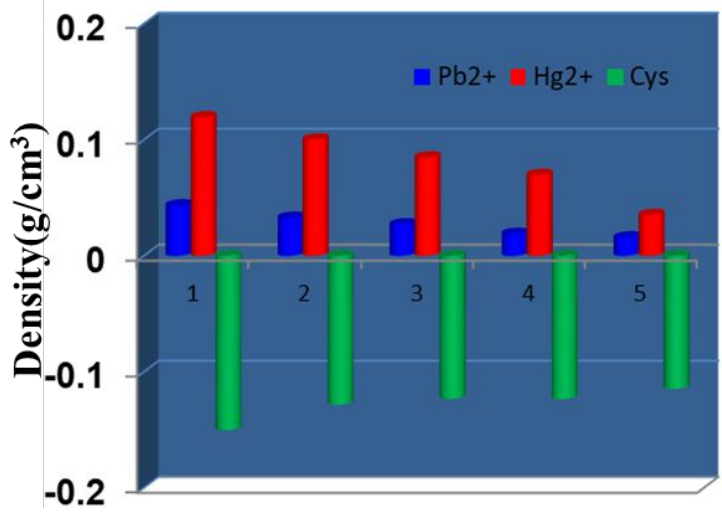

Fig. S6 Reversibility evaluation of the proposed system. The density changes of AS1411 after introducing $\mathrm{Pb}^{2+}, \mathrm{Hg}^{2+}$, Cys for 5 times in succession. 